\title{
Metastatic Cutaneous Squamous Cell Carcinoma in Liver Successfully Treated With Partial Hepatectomy and Adjuvant Irinotecan Chemotherapy
}

\author{
YASUHIKO ASAHINA, YOSHIO NAKAMURA, KEIJI TANESE, IKUKO HIRAI, \\ KEITARO FUKUDA, MASAYUKI AMAGAI and TAKERU FUNAKOSHI \\ Department of Dermatology, Keio University School of Medicine, Tokyo, Japan
}

\begin{abstract}
Background: The management of distant metastatic cutaneous squamous cell carcinoma $(c S C C)$ relies mainly on chemotherapies and radiotherapy. Management of patients with cSCC with surgically resectable distant metastatic lesions is not clear. Case Report: A 59-year-old male had $4 \times 10 \mathrm{~cm}$ sized cSCC on the perianal skin with inguinal lymph node metastasis. Surgical resection was performed followed by radiation and adjuvant carboplatin and farmorubicin combination therapy. Six months later, $25 \mathrm{~mm}$-sized solitary metastatic nodule arose on the liver. Base on his favorable overall condition fulfilling the criteria of tumor resectability for the treatment of tumors in liver and having good performance status, laparoscopic partial hepatectomy and six courses of adjuvant irinotecan therapy were performed. The surgical margin was negative and the patient has maintained complete remission for over 3 years. Conclusion: The clinical course of the present case suggests that surgical approaches should also be considered as candidate additional therapy for resectable distant metastatic cSCC.
\end{abstract}

Cutaneous squamous cell carcinoma (cSCC) is the second most common type of skin cancer, which accounts for approximately $20 \%$ of non-melanoma skin cancer. It arises from the malignant transformation of keratinocytes in the epidermis. Risk factors causing cSCC include exposure to ultraviolent radiation, ionizing radiation, chemical carcinogen exposure, chronic wounds or scars, immuno-suppression and infection with certain

This article is freely accessible online.

Correspondence to: Yoshio Nakamura, Department of Dermatology, Keio University School of Medicine, 35 Shinanomachi, Shinjukuku, Tokyo 160-8582, Japan. Tel: +81 333531211, Fax: +81 333516880, e-mail: yn1109@z5.keio.jp

Key Words: Cutaneous squamous cell carcinoma, liver metastasis, partial hepatectomy, adjuvant chemotherapy, complete remission. genotypes of human papillomavirus $(1,2)$. The incidence rate of cSCC continues to increase worldwide. In the United States of America, it had increased by $263 \%$ from 2000 to 2010 , and has now become a substantial economic burden (3).

Metastasis is rare in cSCC and generally takes favorable clinical course with local surgical resection (4). However, once distant metastasis occurs, it becomes life-threating. Of all cases of metastasis, around $20 \%$ cause distant metastasis in multiple organs, including the lungs, liver, brain, bones, and skin (1). According to the literature reported in 2015, the median progression-free and overall survival of patients with stage IV cSCC were reported to be 0.67 and 2.19 years, respectively, and the 5-year survival rate was $26 \%$ (5). For its treatment, multimodality approaches are recommended by the National Comprehensive Cancer Network guidelines (6). However, current management of distant metastatic cSCC is reliant mainly on chemotherapy and radiotherapy. Evidence for the results of surgical resection of metastatic lesion are limited. Here we report a case of metastatic cSCC in liver successfully treated with surgical resection and adjuvant chemotherapies.

\section{Case Report}

A 59-year-old Chinese male noticed an enlarging tumor on the perianal skin of 8 months duration and presented to our clinic. He had past history of human immunodeficiency virus infection which had been well controlled with combined antiretroviral therapy administrating emtricitabine/tenofovir disoproxil fumarate, darunavir ethanolate and ritonavir.

Clinically, a mushroom shaped, $4 \times 10 \mathrm{~cm}$ exophytic tumor was noted. The tumor surface was eroded and bled easily (Figure 1A). Histopathological findings of the incisional biopsy showed proliferation of atypical keratinocytes. Diagnosis of cSCC was made and palliative surgical resection was performed. Histopathologically, irregularly shaped tumor nests invaded deeply into the dermis (Figure 1B). Tumor nests were composed of atypical keratinocytes with 


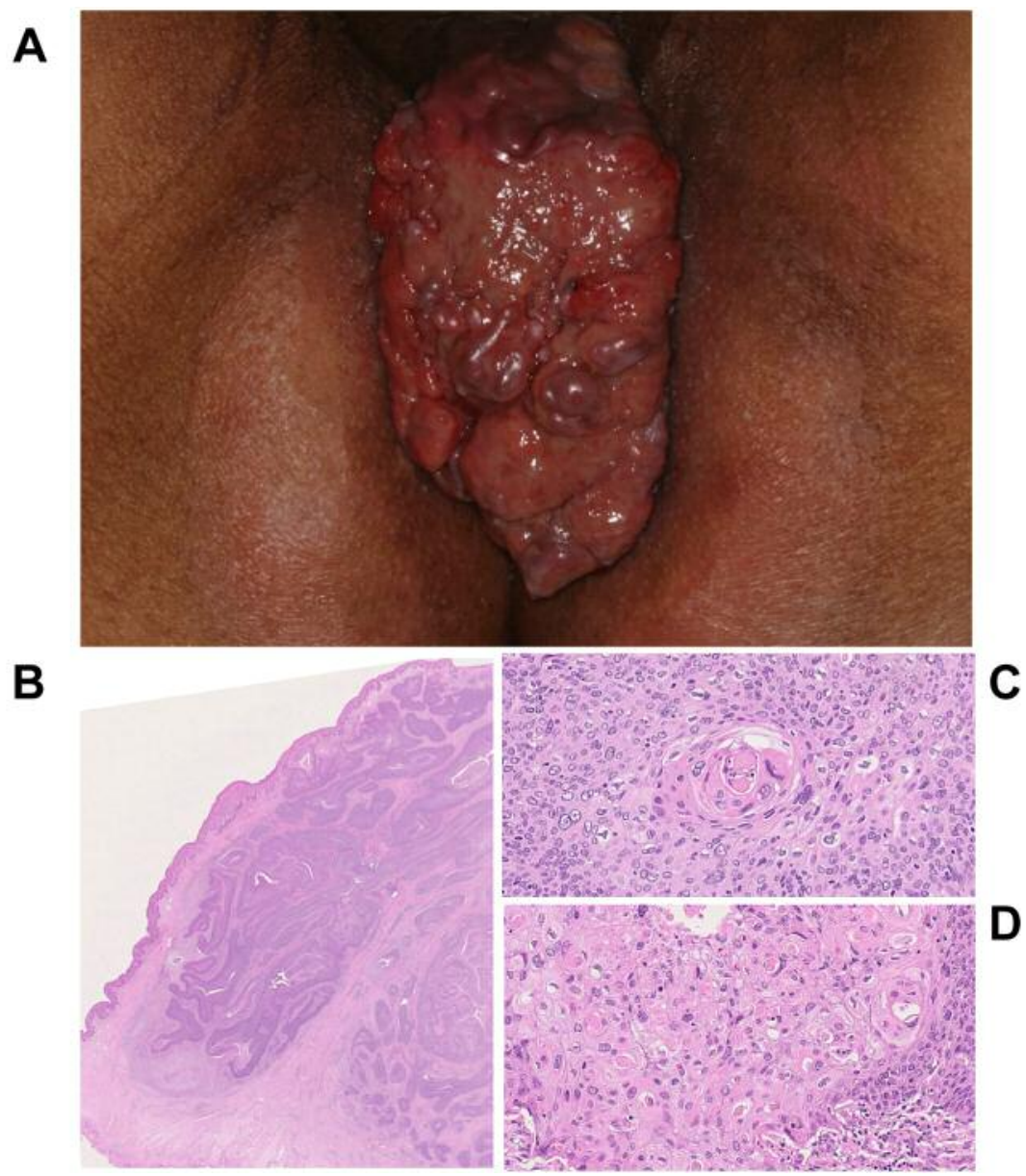

Figure 1. Clinical presentation of the primary lesion. A: A 4x10 cm, enlarging exophytic tumor on the perianal skin had been noticed for 8 months. The tumor surface was eroded and bled easily. B: Representative histopathological aspect of the primary lesion. Hematoxylin and eosin staining. Magnification, $\times 1$. Irregular-shaped basophilic tumor nests deeply invaded into the dermis. C: High-power view of B. Magnification, $\times 100$. Several tumor nests contained a keratin pearl. D: High-power magnification of B. Magnification, x200. Tumor nests were composed of atypical keratinocytes with hyperchromatic and clumped nuclei. Individual cell keratinization was also noted.

hyperchromatic nuclei, and several tumor nests contained keratin pearl (Figure 1C and D). As inguinal lymph node metastasis was detected by ${ }^{18} \mathrm{~F}$-fluorodeoxyglucose positronemission tomography, further radiotherapy for the primary and inguinal lesions followed by five courses of carboplatin and farmorubicin combination therapy were performed.

Six months later, magnetic resonance imaging demonstrated shrinkage of inguinal lymph node metastasis, yet a $25 \mathrm{~mm}$ sized solitary well demarcated nodule was found on the liver (Figure 2A). Computed tomography-guided biopsy of the nodule revealed aberrant proliferation of atypical keratinocytes with nuclear atypia, and diagnosis of metastatic CSCC was made (Figure 2B). The metastatic lesion on the liver was solitary and well demarcated. Patient's Eastern Cooperative Oncology Group performance status was 0 and liver function was normal. Based on these findings, the patient was evaluated as being in an operable condition and further treatment of laparoscopic partial hepatectomy was performed. The surgical margin of the lesion was negative (Figure 2C). After six courses of adjuvant irinotecan administration, the patient has maintained complete remission for over 3 years.

\section{Discussion}

Efficacies of multiple chemotherapeutic agents have been reported for the treatment of advanced $\operatorname{cSCC}(7,8)$, yet the evidence levels provided were poor due to the limitation of them being trials. Nevertheless, recent advances in immunotherapy are providing novel high-evidence therapeutic approaches. Programmed death-1 antibody cemiplimab led to a promising response rate of $46.7 \%$ (35/75 cases) in clinical trials for patients with metastatic $\operatorname{cSCC}(9)$, 


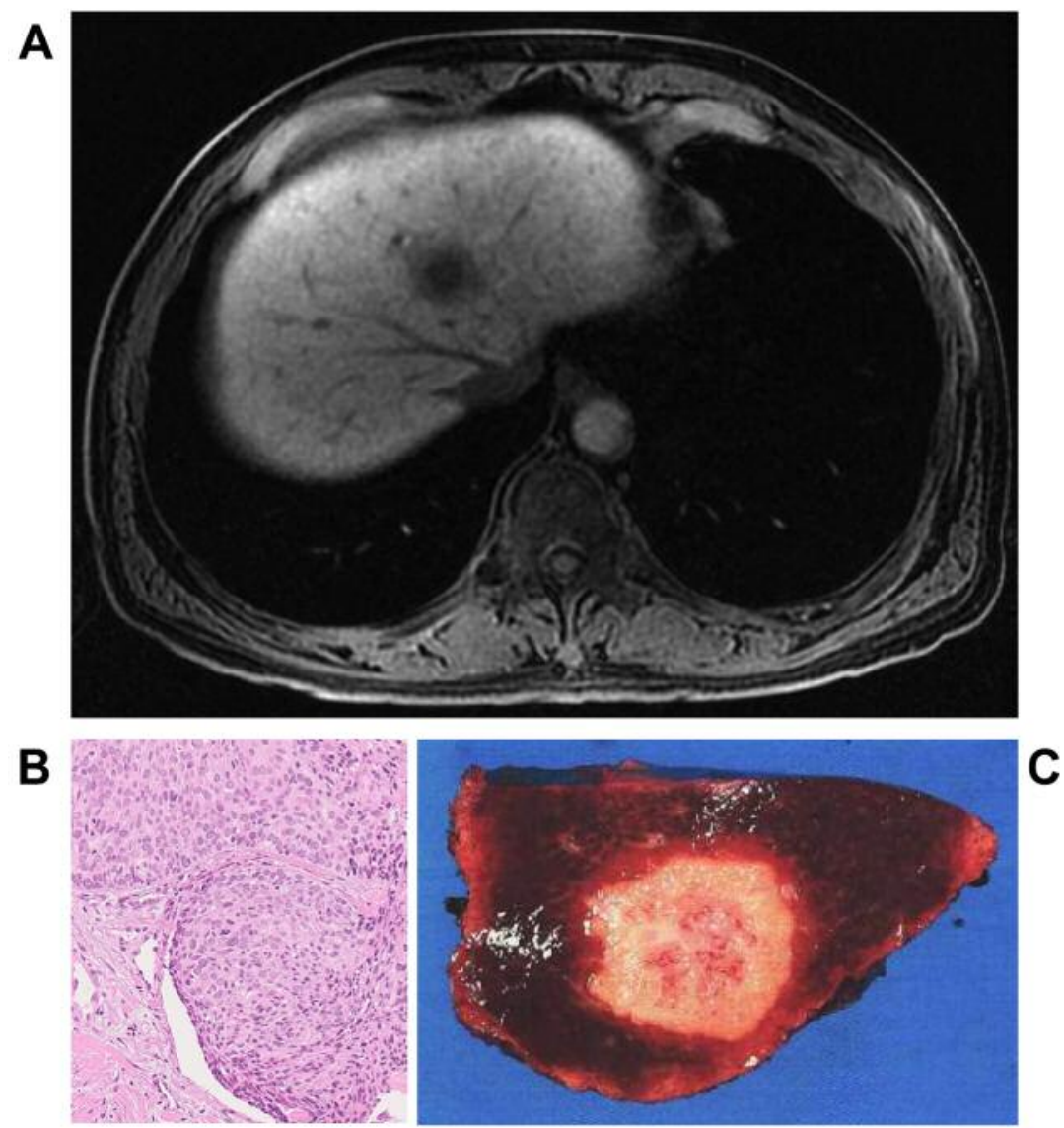

Figure 2. A: Magnetic resonance image. Axial T1-weighted gadolinium ethoxybenzyl diethylenetriamine penta-acetic acid-enhanced hepatobiliary phase. A hypointensity lesion with peripheral rim enhancement was noted in the liver (arrow). B: Histopathological findings of the hepatic nodule obtained by computed tomography-guided biopsy. Hematoxylin and eosin staining. Aberrant proliferation of atypical keratinocytes with nuclear atypia is apparent. Magnification, $\times 100$. B: Macroscopic features of the liver resected by partial hepatectomy. A solitary, well-demarcated nodule was noted in the resected liver. The surgical margin of the tumor was negative.

and was consequently approved by the U.S. Food and Drug Administration in 2018. However, a limited number of patients achieve complete response, and the majority of patients with distant metastatic cSCC still have to live with residual tumor lesion. Therefore, further therapeutic options are required to improve those outcomes.

In our case, liver metastasis arose after adjuvant carboplatin and farmorubicin combination therapy for the inguinal lesions, hence additional treatment was required. Among several therapeutic options, partial hepatectomy and subsequent adjuvant irinotecan administration were adopted based on the following reasons.

First of all, metastasis was isolated in the liver and the lesion was solitary and well demarcated. For the treatment of cancer-bearing patients whose metastatic lesion is isolated in the liver, utilization of several liver-directed therapies is considered, including surgical resection, hepatic arterial infusion therapy and transcatheter arterial chemoembolization.
In fact, hepatectomy has been reported to prolong the prognoses of patients with liver metastasis of colorectal cancer (10). The Hepato-Pancreato-Biliary Expert Consensus Statement 2012 proposed criteria of tumor resectability for the treatment of tumors in liver. It includes expectation of a margin-negative resection, adequate future liver remnant volume, and amenability of venous resection or reconstruction (11). As this case not only fulfilled all of these criteria but also had normal liver function, it was considered to be eligible for partial hepatectomy. Secondly, the patient's Eastern Cooperative Oncology Group performance status was 0, and he was expected to be able to tolerate not only surgery but also adjuvant chemotherapy. Irinotecan is approved for the treatment of cSCC by the Japanese Ministry of Health Labor and Welfare based on the result of a phase II trial conducted in Japan for patients with $\mathrm{cSCC}$ and melanoma. It led to a response rate of $39.4 \%$ (13/33) for Japanese patients with locally advanced or metastatic cSCC including two complete 
and 11 partial responses. Furthermore, seven out of 11 patients with partial response were able to undergo complete tumor resection after the treatment. Regarding safety concerns, $56.6 \%$ (43/76) had leukopenia, 52.6\% (40/76) anorexia, and $47.4 \%(36 / 76)$ nausea/vomiting noted as grade 2 or higher adverse events among both cSCC and melanoma patients (7). While these adverse reactions were well tolerable and reversible, irinotecan is now administered carefully after confirming laboratory data and patient conditions. Thirdly, the pathological tumor differentiation status was well to moderate, which was expected to be less aggressive. These favorable overall patient conditions and pathological features of the tumor led the good postsurgical outcome for this patient.

\section{Conclusion}

While the current therapeutic strategy for distant metastatic cSCC relies mainly on chemotherapy and radiotherapy, the clinical course of the present case suggests that surgical resection of metastatic lesions is also a therapeutic option dependent on the overall patient condition and dispersion status of the metastatic lesion. If a metastasis is isolated in the liver and the lesion is solitary and well demarcated, liverdirected therapies including partial hepatectomy should be considered as a candidate additional therapy. Accumulation of further evidence is desired in order to establish the clinical positioning of surgical resection in therapy of distant metastatic cSCC.

\section{Conflicts of Interest}

The Authors have nothing to declare.

\section{Authors' Contributions}

Y.A. reviewed the past literature and wrote the article with support from Y.N. and K.T. T.F. led examinations and decided the treatment policy. K.T. and Y.N. diagnosed pathologically. Y.N. and I.H. performed surgical treatment. T.F., Y.N. and I.H. contributed to the management of the patients. M.A, K.F. and T.F encouraged Y.A. and supervised the work. Y.N. was responsible for the organisation and coordination of this work. All Authors contributed to the writing of the final article.

\section{References}

1 Alam $\mathrm{M}$ and Ratner D: Cutaneous squamous-cell carcinoma. N Engl J Med 344(13): 975-983, 2001. PMID: 11274625. DOI: 10.1056/NEJM200103293441306

2 Fears T, Scotto J and Schneiderman M: Mathematical models of age and ultraviolet effects on the incidence of skin cancer among whites in the United States. Am J Epidemiol 105(5): 420-427, 1977. PMID: 860705.
3 Muzic JG, Schmitt AR, Wright AC, Alniemi DT, Zubair AS, Olazagasti Lourido JM, Sosa Seda IM, Weaver AL and Baum CL: Incidence and trends of basal cell carcinoma and cutaneous squamous cell carcinoma: a population-based study in Olmsted County, Minnesota, 2000 to 2010. Mayo Clin Proc 92(6): 890898, 2017. PMID: 28522111. DOI: 10.1016/j.mayocp.2017.02.015

4 Schmults CD, Karia PS, Carter JB, Han J and Qureshi AA: Factors predictive of recurrence and death from cutaneous squamous cell carcinoma: A 10-year, single-institution cohort study. JAMA Dermatol 149(5): 541-547, 2013. PMID: 23677079. DOI: 10.1001/jamadermatol.2013.2139

5 Zhu GA and Chang ALS: Overall and progression-free survival of stage 4 cutaneous squamous cell carcinoma at a single large referral center. J Am Acad Dermatol 73(1): 165-166, 2015. PMID: 26089053. DOI: 10.1016/j.jaad.2015.03.028

6 NCCN guidelines in Oncology: Squamous Cell Skin Cancer 2018. Available at: https://www.nccn.org/professionals/ physician_gls/pdf/squamous.pdf

7 Ikeda S, Ishihara K, Oura T, Takahashi M, Ohtuka F, Tamaki K, Kitamura K, Yanagawa S, Ishibashi Y, Morishima T, Takahashi H, Ohhara K, Nakajima H, Saida T, Mori S, Matsunaka M, Arata J, ShimanoS, Yamamoto S, Hori Y, Nonaka S, Ogawa N and Taguchi T: Phase II study of camptothecin in patients with squamous cell carcinoma of the skin. Skin Cancer 8(3): 503-513, 1993. DOI: $10.5227 /$ skincancer.8.503

8 Guthrie TH Jr, Porubsky ES, Luxenberg MN, Shah KJ, Wurtz KL and Watson PR: Cisplatin-based chemotherapy in advanced basal and squamous cell carcinoma of the skin: results in 28 patients including 13 patients receiving multimodality therapy. J Clin Oncol 8(2): 342-346, 1990. PMID: 2405109. DOI: 10.1200/LCO.1990.8.2.342

9 Migden MR, Rischin D, Schmults CD, Guminski A, Hauschild A, Lewis KD, Chung CH, Hernandez-Aya L, Lim AM, Chang ALS, Rabinowits G, Thai AA, Dunn LA, Hughes BGM, Khushalani NI, Modi B, Schadendorf D, Gao B, Seebach F, Li S, Li J, Mathias M, Booth J, Mohan K, Stankevich E, Babiker HM, Brana I, GilMartin M, Homsi J, Johnson ML, Moreno V, Niu J, Owonikoko TK, Papadopoulos KP, Yancopoulos GD, Lowy I and Fury MG: PD-1 blockade with cemiplimab in advanced cutaneous squamouscell carcinoma. N Engl J Med 379(4): 341-351, 2018. PMID: 29863979. DOI: 10.1056/NEJMoa1805131

$10 \mathrm{Xu} \mathrm{F}$, Tang B, Jin TQ and Dai CL: Current status of surgical treatment of colorectal liver metastases. World J Clin Cases 6(14): 716-734, 2018. PMID: 30510936. DOI: 10.12998/wjcc.v6.i14.716

11 Adams RB, Aloia TA, Loyer E, Pawlik TM, Taouli B, Vauthey JN; Americas Hepato-Pancreato-Biliary Association; Society of Surgical Oncology; Society for Surgery of the Alimentary Tract: Selection for hepatic resection of colorectal liver metastases: Expert Consensus statement. HPB 15(2): 91-103, 2013. PMID: 23297719. DOI: $10.1111 / \mathrm{j} .1477-2574.2012 .00557 . \mathrm{x}$

Received November 13, 2019

Revised November 29, 2019

Accepted December 3, 2019 